\title{
Trough Melatonin Levels Differ between Early and Late Phases of Alzheimer Disease
}

\author{
Chieh-Hsin Lin ${ }^{1,2,3}$, Chih-Chiang Chiu ${ }^{4,5}$, Hsien-Yuan Lane ${ }^{2,6,7}$ \\ ${ }^{1}$ Department of Psychiatry, Kaohsiung Chang Gung Memorial Hospital, Chang Gung University College of Medicine, Kaohsiung, ${ }^{2}$ Graduate \\ Institute of Biomedical Sciences, China Medical University, Taichung, ${ }^{3}$ School of Medicine, Chang Gung University, Taoyuan, ${ }^{4}$ Department \\ of Psychiatry, Taipei City Psychiatric Center, ${ }^{5}$ Department of Psychiatry, School of Medicine, Taipei Medical University, Taipei, ${ }^{6}$ Department \\ of Psychiatry and Brain Disease Research Center, China Medical University Hospital, ${ }^{7}$ Department of Psychology, College of Medical and Health \\ Sciences, Asia University, Taichung, Taiwan
}

Objective: Melatonin has been considered to have an essential role in the pathophysiology of Alzheimer's disease (AD) for its regulatory function on circadian rhythm and interaction with glutamate for the modulation of learning and memory. Previous studies revealed that melatonin levels decreased in patients with AD. However, melatonin supplement didn't show promising efficacy for AD. This study compared trough melatonin levels among elderly people with different severities of cognitive deficits.

Methods: We enrolled 270 elder individuals (consisting four groups: healthy elderly, amnestic mild cognitive impairment $[\mathrm{MCl}]$, mild $\mathrm{AD}$, and moderate-severe $\mathrm{AD}$ ) in the learning cohort. Trough melatonin levels in plasma were measured using ELISA. Cognitive function was evaluated by Clinical Dementia Rating Scale (CDR) and Mini-Mental State Examination (MMSE). An independent testing cohort, also consisting of four groups, was enrolled for ascertainment. Results: In the learning cohort, trough melatonin levels decreased in the $\mathrm{MCl}$ group but elevated in the mild and moderate to severe $\mathrm{AD}$ groups. Trough melatonin levels were associated with CDR and MMSE in $\mathrm{MCl}$ or $\mathrm{AD}$ patients significantly. In the testing cohort, the results were similar to those in the learning cohort.

Conclusion: This study demonstrated that trough melatonin levels in the peripheral blood were decreased in $\mathrm{MCl}$ but increased with the severity of AD. The finding supports the trials indicating that melatonin showed efficacy only in $\mathrm{MCl}$ but not in $\mathrm{AD}$. Whether trough melatonin level has potential to be a treatment response biomarker for $\mathrm{AD}$, especially its early phase needs further studies.

KEY WORDS: Melatonin; Alzheimer's disease; Mild cognitive impairment; Cognitive function; Clinical dementia rating scale; Mini-mental state examination.

\section{INTRODUCTION}

Cognitive deterioration is prevalent in the elderly population, particularly in the aging society. Alzheimer's disease (AD) is the most common cause of cognitive deterioration in the elderly. Early detection and intervention are vital for preventing or slowing the development of AD, because the brain changes in pathology are found years or

Received: May 7, 2020 / Revised: July 22, 2020

Accepted: July 30, 2020

Address for correspondence: Hsien-Yuan Lane

Department of Psychiatry, China Medical University Hospital, No.

2, Yuh-Der Road, Taichung 404, Taiwan

E-mail: hylane@gmail.com

ORCID: https://orcid.org/0000-0003-2162-8174 even decades before the cognitive and functional decline in $\mathrm{AD}$ patients [1].

Currently, the diagnosis of mild cognitive impairment $(\mathrm{MCl}$, a condition with minimal cognitive impairment and relatively intact daily functioning) [2] or AD is based upon symptomatology. Feasible laboratory tests for assisting the diagnosis or predicting treatment response are still lacking, particularly those from peripheral approach. However, collecting sample from brain tissue is difficult and relatively more invasive. It has been proposed that gene expression in the peripheral blood might be a surrogate for that in the brain when the target gene is expressed simultaneously in peripheral and central tissues [3].

Many possible pathophysiological mechanisms have

(c) This is an Open-Access article distributed under the terms of the Creative Commons Attribution Non-Commercial License (http://creativecommons.org/licenses/by-nc/4.0) which permits unrestricted non-commercial use, distribution, and reproduction in any medium, provided the original work is properly cited. 
been proposed for $A D$, dysregulation of melatonin is among them [4]. Melatonin is involved in the synchronization of the circadian rhythm [5] and has a role as an antioxidant [6]. It has been well-known that glutamate modulation involves in the melatonin synthesis [7-9]. Glutamate is the most abundant amino acid neurotransmitter in the mammalian brain, and its dysfunction contributes to various neuropsychiatric disorders. On the other hand, melatonin also has protective effect against glutamate-induced excitotoxicity which may result in neurodegeneration $[10,11]$.

Some previous studies suggest that the melatonin level decreases in AD patients [12-14] or daily rhythm of serum melatonin disappears in patients with neurodegenerative type dementias [15]. However, the efficacy of melatonin to treat patients with $\mathrm{AD}$ was not verified in clinical trials. Interestingly, there is evidence to show therapeutic effect of melatonin on patients with $\mathrm{MCl}[16,17]$. Moreover, a large-scale review found no evidence that melatonin or ramelteon (an antagonist for $\mathrm{MT}_{1}$ and $\mathrm{MT}_{2}$ receptors) helped sleep problems in patients with moderate to severe AD [18]. These evidences suggest that the changes of melatonin might not be the same among different stages of dementia, and the role of melatonin needs to be further investigated in different stages of $A D$.

Identifying risky population (e.g., elderly people with lower melatonin) might be helpful in preventing and treating AD. The melatonin level peaks between midnight and early morning [19]. Measuring trough melatonin level in the morning might be more feasible than peak melatonin or total melatonin secretion for large-scale screening in the elderly people. To our knowledge, studies that compare melatonin levels among different stages of $A D$ are lacking. The aim of this study is to examine the trough melatonin levels in elderly healthy individuals and patients with cognitive decline, and investigate the association between trough melatonin levels and the severity of cognitive deficits.

\section{METHODS}

\section{Participants}

All participants were recruited and evaluated from Kaohsiung Chang Gung Memorial Hospital, China Medical University Hospital, and Taipei City Municipal Hospital in Taiwan. This study was carried out in accordance with the recommendations of Good Clinical Practice (GCP), Institutional Review Boards of Kaohsiung Chang Gung Memorial Hospital, China Medical University Hospital, and Taipei City Municipal Hospital, Taiwan. The protocol was approved by the Institutional Review Boards of the aforementioned hospitals (no. 104-9692B, CMUH103REC3-111). All subjects gave written informed consent in accordance with the Declaration of Helsinki.

All participants were $50-100$ years old Han Chinese who were healthy physically with normal blood routine and biochemical tests. All participants were evaluated by research physicians thoroughly. Participants were recruited if they (1) had adequate education for effective communication and were able to complete the assessments of the study, and (2) agreed to join the study and provided written informed consent. Exclusion criteria included major medical, neurological, or psychiatric conditions other than $\mathrm{AD}$; delirium symptoms; substance abuse or dependence (including alcohol); Hachinski Ischemic Score $>4$; history of significant cerebrovascular disease; severe hearing or visual impairment; and being unable to follow protocol. The healthy volunteers were free from any psychiatric disorder. Similarly, all healthy participants had no substance abuse or dependence (including alcohol) diagnosed by the Diagnostic and Statistical Manual of Mental Disorders 4th edition.

- Patients with $\mathrm{MCl}$ satisfied criteria for amnestic $\mathrm{MCl}$ [20] of a probably degenerative course defined as subjective memory complaint and insufficient global cognitive and functional impairment to meet National Institute of Neurological and Communicative Disorders and Stroke and the Alzheimer's Disease and Related Disorders Association (NINCDS-ADRDA) [21] criteria with a Clinical Dementia Rating (CDR) [22] score of 0.5 .

- Patients with mild AD satisfied NINCDS-ADRDA criteria for probable AD with a CDR score of 1.

- Patients with moderate to severe AD satisfied NINCDS-ADRDA criteria for probable AD with a CDR score of 2 or greater.

- Healthy individuals had a CDR score of 0 .

All AD patients were enrolled from the outpatient department at the aforementioned hospitals. All healthy individuals were enrolled from the communities in southern, central and northern Taiwan.

$A D$ patients with and without anti-dementia drugs were 
both recruited. AD patients without anti-dementia drugs were free from those medications for three months or longer. For patients with anti-dementia drugs treatment, those medications had been maintained for three months or longer with unchanged dose. Medication history was determined by reviewing medical records, contacting other health care providers, and interviewing the participants and their family or caregivers. Healthy individuals were free from anti-dementia drugs.

A learning cohort composed of participants with different severities of cognitive deficits was enrolled. In order to specify the effects of age, sex and education on cognitive function and to check whether the finding in the learning cohort can be replicated, we enrolled an independent testing cohort. In the testing cohort, age, sex and educational level were matched among all the groups with different cognitive function.

\section{Cognitive Function Assessments}

The cognitive function of the participants was evaluated by CDR and Mini-Mental State Examination (MMSE) [23]. MMSE is a common tool for the screening of dementia and the measurement of cognition [23]. Nevertheless, MMSE is easily influenced by education and age [24], and has low sensitivity for mild cognitive deficits that limit its use [25].

In contrast, CDR has good discrimination power for dementia with slight impairment [26]. In addition, CDR has shown good reliability and validity for the assessment and staging of dementia with favorable inter-rater reliability [27]. Thus, the grouping of participants was based upon CDR and MMSE that represent both the cognitive and global impairment of the participants. Of note, CDR score is not influenced by education and age [28].

\section{Laboratory Assessments}

\section{Trough melatonin level measurement}

The blood specimens were collected during 8-12 AM for measuring the trough melatonin level. We have collected plasma samples from several subjects for measuring melatonin level at different time points to validate the method. Melatonin protein concentrations were measured using commercially available enzyme-linked immunosorbent assay (ELISA) kits according to the manufacture's recommended protocol (MyBioSource, San
Diego, CA, USA). Briefly, $100 \mu$ plasma samples and the standard were added to each well of a 96-well plate. The solutions were incubated for 2 hours at $37^{\circ} \mathrm{C}$. The liquid was then removed. One hundred microliters of Biotin-antibody $(1 \times)$ was added to each well and incubated for 1 hour at $37^{\circ} \mathrm{C}$. Each well was washed with buffer for 3 times. One hundred microliters of HRP-avidin $(1 \times)$ was added to each well and incubated for 1 hour at $37^{\circ} \mathrm{C}$. Each well was washed with buffer for 5 times and then incubated with $90 \mu$ substrate solution for $15-30$ minutes at $37^{\circ} \mathrm{C}$ with the protection from light. Fifty microliters of stop solution was added to each well, and mixed thoroughly. A Benchmark Plus Microplate Reader (Bio-Rad, Hercules, CA, USA) was used to read the optical density at $450 \mathrm{~nm}$. The concentrations of melatonin in the samples were determined according to a standard curve.

\section{Statistical Analysis}

All participants' clinical and demographic characteristics and melatonin levels were shown as number (percentage) or mean \pm standard deviation. All percentages between groups were compared by $\chi^{2}$ test, and mean values between groups were compared by independent $t$ test or Mann - Whitney $U$ test for two groups, one-way ANOVA or Kruskal - Wallis test for three or more groups. Multiple linear regression analysis was used to generate predictive models for cognitive function. A $p$ value less than 0.05 was considered significant statistically. All statistical analyses were performed using IBM SPSS Statistics version 22.0 (IBM Co., Armonk, NY, USA).

\section{RESULTS}

A total of 270 subjects were enrolled for the learning cohort: 73 healthy individuals, 47 amnestic MCl patients, 98 mild AD patients, and 52 moderate to severe AD patients. There were more males in the controls than the other three $\mathrm{AD}$ groups $(p=0.002)$. The age distribution and education were significantly different among the four groups ( $p<0.001$ ). The percentage of patients using anti-dementia agents (including memantine and AChEI) among the three groups with cognitive deficits reached a borderline significance $(p=0.058)$. The demographic characteristics are shown in Table 1.

We further enrolled an independent age-, sex-, and education-matched cohort (testing cohort) to verify the re- 
Table 1. Demographic characteristics of the learning cohort $(n=270)$

\begin{tabular}{|c|c|c|c|c|c|}
\hline $\begin{array}{l}\text { Demographic } \\
\text { characteristics }\end{array}$ & $\begin{array}{l}\text { Healthy elderly } \\
\qquad(n=73)\end{array}$ & $\begin{array}{c}\mathrm{MCl} \\
(\mathrm{n}=47)\end{array}$ & $\begin{array}{l}\text { Mild AD } \\
(\mathrm{n}=98)\end{array}$ & $\begin{array}{l}\text { Moderate to severe } \\
\qquad A D(n=52)\end{array}$ & $p$ value \\
\hline \multicolumn{6}{|l|}{ Demographics } \\
\hline Sex, female & $31(42.5)$ & $29(61.7)$ & $66(67.3)$ & $37(71.2)$ & $0.002^{\mathrm{a}}$ \\
\hline Age (yr) & $66.3 \pm 9.0$ & $66.1 \pm 5.0$ & $75.2 \pm 7.1$ & $82.5 \pm 7.0$ & $<0.001^{b}$ \\
\hline CDR & $0.0 \pm 0.0$ & $0.5 \pm 0.0$ & $1.0 \pm 0.0$ & $2.4 \pm 0.5$ & $<0.001^{\mathrm{b}}$ \\
\hline MMSE & $28.3 \pm 1.5$ & $23.1 \pm 3.2$ & $18.6 \pm 4.2$ & $10.5 \pm 4.0$ & $<0.001^{b}$ \\
\hline Education (yr) & $13.6 \pm 2.3$ & $4.5 \pm 4.3$ & $3.6 \pm 2.7$ & $3.3 \pm 3.7$ & $<0.001^{b}$ \\
\hline \multicolumn{6}{|c|}{ Number of subjects using anti-dementia drugs } \\
\hline Total & NA & $4(8.5)$ & $25(25.5)$ & $11(21.2)$ & $0.058^{\mathrm{a}, *}$ \\
\hline Donepezil (dose) & NA & $7.5 \pm 2.9(n=4)$ & $9.2 \pm 1.9(n=18)$ & $9.3 \pm 1.9(n=7)$ & $0.280^{\mathrm{a}, *}$ \\
\hline Rivastigmine (dose) & NA & 0 & $6.8 \pm 2.6(n=4)$ & $9.0(n=1)$ & $0.325^{\mathrm{a}, *}$ \\
\hline Galantamine (dose) & NA & 0 & $16.0 \pm 0.0(n=3)$ & $16.0(n=1)$ & $0.472^{\mathrm{a}, *}$ \\
\hline Memantine (dose) & NA & 0 & 0 & $20.0 \pm 0.0(n=2)$ & $0.060^{\mathrm{a}, *}$ \\
\hline Melatonin level (pg/ml) & $92.9 \pm 53.6$ & $55.3 \pm 49.7$ & $71.5 \pm 49.9$ & $115.7 \pm 60.1$ & $<0.001^{\mathrm{b}}$ \\
\hline $\begin{array}{l}\text { Melatonin level }(\mathrm{pg} / \mathrm{ml}) \text { in subjects } \\
\text { using anti-dementia drugs }\end{array}$ & NA & $57.5 \pm 78.5$ & $74.5 \pm 52.2$ & $112.0 \pm 61.0$ & $0.138^{b_{1 *}}$ \\
\hline $\begin{array}{l}\text { Melatonin level }(\mathrm{pg} / \mathrm{ml}) \text { in subjects } \\
\text { without anti-dementia drugs }\end{array}$ & $92.9 \pm 53.6$ & $55.1 \pm 47.6$ & $70.5 \pm 49.5$ & $116.7 \pm 60.6$ & $<0.001^{\mathrm{b}}$ \\
\hline
\end{tabular}

Values are presented as number (\%) or mean \pm standard deviation.

AD, Alzheimer's disease; MCl, mild cognitive impairment; CDR, Clinical Dementia Rating; MMSE, Mini Mental Status Examination; NA, not associated.

${ }^{\mathrm{a}}$ Chi-square test; ${ }^{\mathrm{b}}$ Kruskal - Wallis test; *Comparison among $\mathrm{MCl}$, mild $\mathrm{AD}$ and moderate to severe $\mathrm{AD}$ groups.

Table 2. Demographic characteristics of the testing cohort (age-, sex- and education-matched) $(n=136)$

\begin{tabular}{|c|c|c|c|c|c|}
\hline $\begin{array}{l}\text { Demographic } \\
\text { characteristics }\end{array}$ & $\begin{array}{l}\text { Healthy elderly } \\
\qquad(\mathrm{n}=35)\end{array}$ & $\begin{array}{c}\mathrm{MCl} \\
(\mathrm{n}=34)\end{array}$ & $\begin{array}{l}\text { Mild AD } \\
(\mathrm{n}=36)\end{array}$ & $\begin{array}{l}\text { Moderate to severe } \\
\qquad \text { AD }(n=31)\end{array}$ & $p$ value \\
\hline \multicolumn{6}{|l|}{ Demographics } \\
\hline Sex, female & $18(51.4)$ & $18(52.9)$ & $19(51.8)$ & $16(51.6)$ & $0.999^{\mathrm{a}}$ \\
\hline Age (yr) & $70.0 \pm 10.1$ & $70.5 \pm 9.2$ & $70.3 \pm 9.8$ & $71.5 \pm 8.7$ & $0.935^{\mathrm{C}}$ \\
\hline CDR & $0.0 \pm 0.0$ & $0.5 \pm 0.0$ & $1.0 \pm 0.0$ & $2.5 \pm 0.5$ & $<0.001^{\mathrm{b}}$ \\
\hline MMSE & $27.8 \pm 1.6$ & $23.6 \pm 3.1$ & $18.1 \pm 5.0$ & $10.6 \pm 5.4$ & $<0.001^{\mathrm{b}}$ \\
\hline Education (yr) & $8.2 \pm 4.4$ & $9.5 \pm 4.2$ & $9.1 \pm 4.8$ & $8.5 \pm 5.4$ & $0.142^{\mathrm{b}}$ \\
\hline \multicolumn{6}{|c|}{ Number of subjects using anti-dementia drugs } \\
\hline Total & NA & $5(14.7)$ & $18(50.0)$ & $5(16.1)$ & $0.001^{\mathrm{a}, *}$ \\
\hline Donepezil (dose) & NA & $6.3 \pm 2.5(n=4)$ & $8.9 \pm 2.2(n=9)$ & $10.0 \pm 0.0(n=2)$ & $0.086^{\mathrm{a}, *}$ \\
\hline Rivastigmine (dose) & NA & $9.0(n=1)$ & $7.5 \pm 2.6(n=3)$ & $6.7 \pm 2.1(n=3)$ & $0.519^{\mathrm{a}, *}$ \\
\hline Galantamine (dose) & NA & 0 & $14.7 \pm 3.3(n=6)$ & 0 & $0.003^{\mathrm{a}, *}$ \\
\hline Memantine (dose) & NA & 0 & 0 & 0 & NA \\
\hline Melatonin level (pg/ml) & $92.9 \pm 51.5$ & $42.7 \pm 38.2$ & $64.5 \pm 51.6$ & $127.8 \pm 48.7$ & $<0.001^{b}$ \\
\hline $\begin{array}{l}\text { Melatonin level (pg/ml) in subjects } \\
\text { using anti-dementia drugs }\end{array}$ & NA & $13.8 \pm 11.6$ & $47.0 \pm 44.5$ & $120.9 \pm 42.2$ & $0.001^{\mathrm{b}, *}$ \\
\hline $\begin{array}{l}\text { Melatonin level }(\mathrm{pg} / \mathrm{ml}) \text { in subjects } \\
\text { without anti-dementia drugs }\end{array}$ & $92.9 \pm 51.5$ & $47.7 \pm 39.1$ & $82.1 \pm 53.3$ & $129.2 \pm 50.5$ & $<0.001^{\mathrm{b}}$ \\
\hline
\end{tabular}

Values are presented as number (\%) or mean \pm standard deviation.

AD, Alzheimer's disease; MCl, mild cognitive impairment; CDR, Clinical Dementia Rating; MMSE, Mini Mental Status Examination NA, not associated.

${ }^{\mathrm{a}}$ Chi-square test; ${ }^{\mathrm{b}}$ Kruskal - Wallis test; ${ }^{\mathrm{c}} \mathrm{ANOVA}$ test; ${ }^{*}$ Comparison among $\mathrm{MCl}$, mild AD and moderate to severe AD groups.

sults in the learning cohort. In the testing cohort, there was no significant difference among the four groups in age, sex, and education $(p=0.935,0.999,0.639$, respectively). There was a significant difference in the percent- age of anti-dementia drugs use in the three groups with cognitive deficits $(p=0.001)$. The demographic characteristics of the testing cohort are shown in Table 2. 


\section{Trough Melatonin Levels were Lowest in Patients with Mild Cognitive Impairment}

The trough melatonin levels in the healthy controls, amnestic $\mathrm{MCl}$, mild $\mathrm{AD}$, and moderate to severe AD were $92.9 \pm 53.6 \mathrm{pg} / \mathrm{ml}, 55.3 \pm 49.7 \mathrm{pg} / \mathrm{ml}, 71.5 \pm 49.9 \mathrm{pg} / \mathrm{ml}$ and $115.7 \pm 60.1 \mathrm{pg} / \mathrm{ml}$, respectively $(p<0.001)$ (Table 1 and Fig. 1A). The melatonin levels in amnestic $\mathrm{MCl}$ or $\mathrm{AD}$ patients $(79.3 \pm 57.2)$ were lower than those in healthy controls $(p=0.078)$. Post-hoc analysis using Bonferroni method revealed that the melatonin level in amnestic $\mathrm{MCl}$ patients was lower than healthy controls and moderate to severe AD patients ( $p<0.001, p<0.001$, respectively). The melatonin level in mild AD patients was lower than moderate to severe AD patients $(p<0.001)$. The melatonin levels between $\mathrm{MCl}$ patients and mild AD patients were not significantly different $(p=0.516)$. The melatonin levels between controls and moderate to severe AD patients were not significantly different either $(p=0.111)$. A borderline significance was found in melatonin level between healthy controls and patients with mild AD ( $p=$ 0.056). The melatonin levels in patients with or without anti-dementia drugs were not significantly different (Table 1).

For the testing cohort, the trough melatonin levels in the healthy individuals, amnestic $\mathrm{MCl}$, mild $\mathrm{AD}$ and moderate to severe $\mathrm{AD}$ were $92.9 \pm 51.5 \mathrm{pg} / \mathrm{ml}, 42.7 \pm 38.2$ $\mathrm{pg} / \mathrm{ml}, 64.5 \pm 51.6 \mathrm{pg} / \mathrm{ml}$ and $127.8 \pm 48.7 \mathrm{pg} / \mathrm{ml}$, respectively ( $p<0.001$ ) (Table 2 and Fig. 1B). The melatonin levels in amnestic $\mathrm{MCl}$ or AD patients $(76.6 \pm 58.2)$ were lower than those in healthy controls $(p=0.144)$. In post-hoc analysis using Bonferroni method, the melatonin level in controls was higher than amnestic $\mathrm{MCl}$ patients and lower than moderate to severe AD patients $(p<$ $0.001, p=0.022$, respectively). The melatonin level in amnestic $\mathrm{MCl}$ patients was lower than healthy controls and moderate to severe AD patients ( $p<0.001, p<$ 0.001 , respectively). The melatonin level in mild AD was lower than moderate to severe AD patients $(p<0.001)$. The melatonin levels were not significantly different between mild AD patients and healthy controls, and between amnestic $\mathrm{MCl}$ and mild $\mathrm{AD}(p=0.082, p=0.355$, respectively). The melatonin levels in mild AD patients receiving anti-dementia drugs were significantly lower than those not receiving anti-dementia drugs $(p=0.039)$. The melatonin levels in amnestic $\mathrm{MCl}$ and moderate to severe AD patients receiving anti-dementia drugs were not significantly different from those without anti-dementia drugs ( $p=0.066, p=0.734$, respectively) (Table 2 ).

We further checked the association between melatonin levels and age in each group of subjects. There were no significant association between melatonin levels and age in healthy individuals, amnestic $\mathrm{MCl}$, mild $\mathrm{AD}$ and moderate to severe AD groups $(p=0.606,0.701,0.308$, 0.414 , respectively). We also checked the association between melatonin levels and anti-dementia drugs use. There was no significant association between melatonin levels and anti-dementia drugs use in all subjects ( $p=$ 0.077).

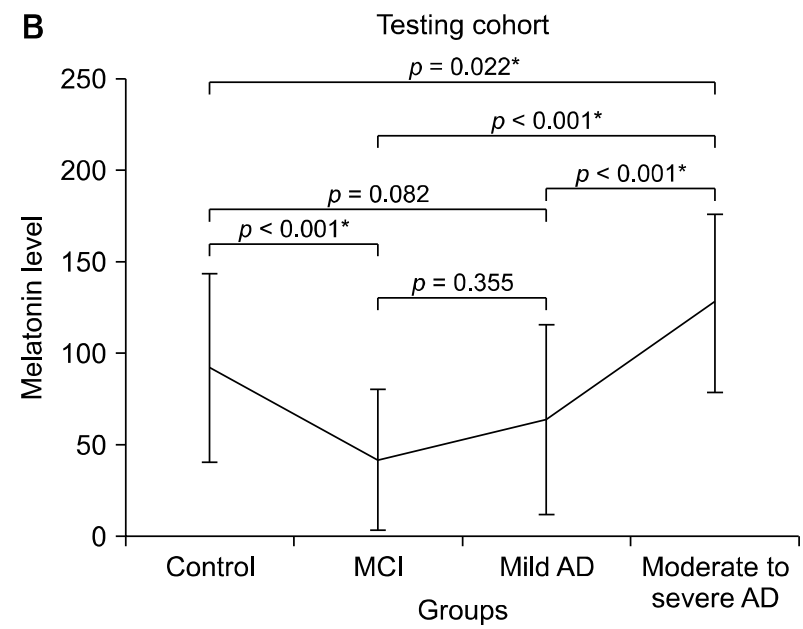

Fig. 1. (A) Learning cohort. (B) Testing cohort. $\mathrm{MCl}$, mild cognitive impairment; AD, Alzheimer's disease. ${ }^{*} p<0.05$.

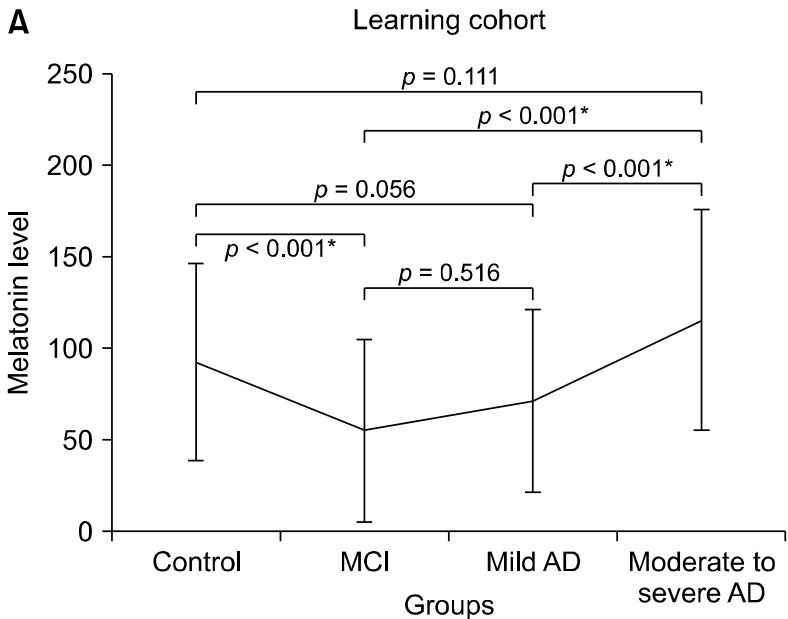

$$
{ }^{*} p<0.05 \text {. }
$$


Table 3. Multiple linear regression analyses of independent factors associated with cognitive function in all participants (learning cohort and testing cohort) (stepwise)

\begin{tabular}{lrrr}
\hline \multicolumn{1}{c}{ Variable } & B (SE) & $\mathrm{t}$ & $p$ value \\
\hline CDR score & & & \\
Age (yr) & $0.026(0.004)$ & 6.174 & $<0.001$ \\
Sex & $-0.042(0.080)$ & 1.082 & 0.280 \\
Education (yr) & $0.354(0.103)$ & 3.445 & 0.001 \\
Anti-dementia drug use & $0.004(0.001)$ & 5.683 & $<0.001$ \\
Melatonin level (pg/ml) & & & \\
Adjusted R square =0.281 & & & \\
MMSE score & $-0.162(0.036)$ & -4.470 & $<0.001$ \\
Age (yr) & $-0.754(0.661)$ & -1.141 & 0.255 \\
Sex & $0.503(0.066)$ & 7.579 & $<0.001$ \\
Education (yr) & $-2.896(0.850)$ & -3.408 & 0.001 \\
Anti-dementia drug use & & & \\
Melatonin level (pg/ml) & $-0.030(0.006)$ & -5.388 & $<0.001$ \\
Adjusted R square $=0.299$ & & & \\
\hline
\end{tabular}

The regression model was adjusted with age, sex, and education. The variables were melatonin level and anti-dementia drug use. CDR, Clinical Dementia Rating; MMSE, Mini Mental Status Examination.

\section{Factors Associated with CDR and MMSE Score}

We applied multiple linear regression analyses for testing the variables that might be related to CDR and MMSE score. The multiple regression models were adjusted with sex, age and educational level. In the overall (learning plus testing) cohort, CDR score was associated with age, education, anti-dementia drug use and melatonin level significantly (adjusted R square $=0.281$ ). Similarly, MMSE score was also associated with age, education, anti-dementia drug use and melatonin level significantly (adjusted R square $=0.299$ ) (Table 3 ). In the learning cohort, CDR was associated with age, education, and melatonin level significantly (adjusted R square $=0.500$ ). MMSE was also significantly associated with age, education, and melatonin level (adjusted R square $=0.525$ ) (Supplementary Table 1; available online). In the testing cohort, CDR was associated with anti-dementia drug use and melatonin level significantly (adjusted R square $=0.150$ ). MMSE was significantly associated with education, anti-dementia drug use and melatonin level (adjusted R square $=0.130$ ) (Supplementary Table 2; available online).

\section{DISCUSSION}

This study reports that peripheral trough melatonin levels of amnestic $\mathrm{MCl}$ patients are lower than those observed in elderly controls and AD patients, and that trough melatonin levels are associated with the severity of cognitive impairment. In general, melatonin production declines with age in adults [29]. Although previous studies showed that melatonin levels were lower in patients with $A D$ than controls and suggested that melatonin deficiency may contribute to the acceleration of aging [30], melatonin levels were not examined in different stages of AD. This study demonstrated that trough melatonin levels were lower in amnestic $\mathrm{MCl}$ and increased with the severity of cognitive deficits in patients with AD.

The beneficial effect of melatonin treatment for $A D$ remains controversial. Srinivasan et al. [31] suggested that melatonin replacement has therapeutic potential for $A D$, particularly at the early phases of the disease and in those with intact melatonin receptors. Wu and Swaab [32] also found that the dysfunction of the pineal melatonin synthesis is responsible for melatonin changes during the early $A D$ stage. Our findings support previous reports that suggest melatonin therapy is beneficial for individuals with $\mathrm{MCI}$ but not $\mathrm{AD}$ [16-18]. This study indicates that patients with amnestic $\mathrm{MCl}$, especially, those who have lower melatonin levels, might be the targeted population for melatonin supplement therapy.

There is a diurnal variation of the melatonin level, with the peak at night and trough during daytime due to the suppression by light $[33,34]$. A faster decline of the melatonin level from the nocturnal peak was found in the elderly people compared to young adults [35]. A recent systematic review suggested that total melatonin production in 24 hours does not change in healthy aging, but the maximal nocturnal peak of melatonin level might decline with aging [36]. In this study, the trough melatonin levels were measured instead of nocturnal peak melatonin. The blood sampling time in this study was during 8-12 AM when the melatonin level was considered to be the trough, making the mean melatonin level in the four groups comparable and representative. We believe that trough melatonin levels measurement in the morning is more feasible for the elderly people and has more potential to be developed as large-scale screening tool. The reference range of melatonin level in peripheral blood has been reported to be $3.83-80.4 \mathrm{pg} / \mathrm{ml}$ at 8:00 AM [37]. In our study, the melatonin level of healthy elderly seemed to be a little bit higher than previous reports. A large-scale study found that the melatonin signaling pathway may be 
different with global variation in sunshine duration [38]. Day light exposure is positively associated with urinary 6-sulfatoxymelatonin excretion in the elderly [39]. Whether the abundant sunshine in Taiwan contributes to the higher melatonin level in this study needs further investigation.

Participants in this study were aged between 50 and 100 years old and most women were postmenopause. Most of the participants were free from medications that can modulate the melatonin secretion including agomelatine [40] and ramelteon [41] by a thorough history taking through reviewing medical records, contacting other health care providers, and interviewing the participants and their family or caregivers. However, the possibility of psychotropic drugs use as well as unusual light exposure that might alter the melatonin level or sleep pattern cannot be completely excluded.

Whether melatonin interacts with anti-dementia drugs remains unclear. An in vitro study found synergistic neuroprotective effects of combined galantamine (an acetylcholinesterase inhibitor) and melatonin against oxidative stress in neuroblastoma cells [42]. An animal study found that melatonin supplementation increased choline acetyltransferase activity in the frontal cortex and hippocampus in a transgenic mouse model of AD [43]. A number of melatonin and anti-dementia drugs hybrids have been synthesized and tested for their effects on neurodegenerative diseases [44]. In the learning cohort of this study, the melatonin levels in AD patients receiving anti-dementia drugs were close to patients not receiving anti-dementia drugs, although there seemed to be a trend in the testing cohort that patients using anti-dementia drugs had lower melatonin level than those without anti-dementia drugs. There was no significant association between melatonin levels and anti-dementia drugs use in all subjects in our study ( $p=0.077)$; however, whether melatonin level is altered by anti-dementia drugs use in AD patients needs further evaluation in future clinical studies.

The higher melatonin level in patients with moderate-severe $A D$ in this study deserves further investigation. Zhou et al. [12] has shown that melatonin levels in post-mortem cerebrospinal fluid (CSF) constantly decreases from $\mathrm{MCl}$ to severe AD. To the best of our knowledge, there is no published study that compares melatonin levels in peripheral blood in different stages of AD. The inconsistent finding between CSF and peripheral blood needs further clarification in future studies.
Increased oxidative stress might play a role underlying processes of aging or neurodegenerative diseases. In an animal study, antioxidant enzymes such as $\mathrm{NAD}(\mathrm{P}) \mathrm{H}$ :quinone oxidoreductase 1 , superoxide dismutase 1 and Sirtuin 1 were found increased in some brain regions in 3xTg-AD mice that expressed behavioral and psychological symptoms in dementia (BPSD)-like symptoms [45]. One of the functions of melatonin is to act as an antioxidant $[6,46]$. It might be likely that melatonin excretion increases in later phase of $\mathrm{AD}$ as a compensatory response for the defense of oxidative stress. This preliminary speculation deserves further studies. Moreover, sleeping patterns are sometimes backwards in moderate-severe AD patients. That might be one of the reasons why trough melatonin levels are rather elevated in the moderate-severe AD group.

This study is limited in several aspects. Firstly, findings from this cross-sectional study cannot be extrapolated to longitudinal follow-up study. Secondly, although an earlier study found that melatonin levels in cerebrospinal fluid samples were similar to or lower than those in plasma by using chromatographic run by high-performance liquid chromatography (HPLC) [47], the peripheral blood-CNS relationship of melatonin still requires investigation in $\mathrm{AD}$ patients. In the meanwhile, using ELISA instead of HPLC for the measurement of melatonin level may be another limitation for this study. Thirdly, as aforementioned, the blood samplings were not at the same time for all participants, although we had restricted the blood sampling time to be within 8-12 AM. Determining the timing of the blood collection for melatonin measurement relative to each individual's sleep-wake and light exposure-estimated trough via a sleep diary or actigraphy for the two weeks preceding the melatonin assessment would be of great help in future study. Fourthly, findings from Han Chinese require replication by other groups in various racial populations. Fifthly, depression scores need to be assessed in future study because depression in elderly people is a risk factor for the conversion of AD. Sixthly, the cognitive measurement in this study only included the total score of CDR and MMSE. More complete cognitive measurements should be included in future studies. Lastly, despite a thorough physical and mental work-up to exclude patients with comorbid mental or organic disorders, it was not possible to exclude with certainty other neurodegenerative diseases (e.g., Huntington's disease and Parkinson's disease) that may alter melatonin levels 
[48-51].

In summary, trough melatonin levels in the peripheral blood decrease in $\mathrm{MCl}$ patients, but increase with the severity of cognitive deficits in patients with $\mathrm{AD}$. It is too early to regard melatonin as a biomarker for cognitive aging. However, trough melatonin level has potential to be a biomarker for high risk population for developing AD [52]. Moreover, baseline melatonin level might be able to serve as a treatment response biomarker for melatonin therapy [53]. Combine multiple potential biomarkers for the assistance of diagnosis of $\mathrm{AD}$ is favorable because neurodegenerative diseases are substantially multifactorial. For instance, melatonin level combined with other potential assessments, e.g., D-amino acid oxidase [54] and $\beta$-amyloid [32] levels, may be a possible approach. In the future, the alteration of melatonin level in the progression from the early phase of $\mathrm{AD}$ to its later phase requires further investigation in prospective longitudinal larger scale studies in patients with different stages of cognitive decline. More importantly, whether melatonin, that has high-safety profiles, can be developed as a treatment for $\mathrm{AD}$, especially in its early phase, needs to be testified in larger-scale randomized controlled trials [46].

\section{Acknowledgments}

This work was funded by Ministry of Science and Technology, Taiwan (MOST 105-2314-B-182A-059-), Kaohsiung Chang Gung Memorial Hospital, Taiwan (NMRPG8F0111, BMRPC04), China Medical University Hospital, Taiwan (DMR-106-099) and Taiwan Ministry of Health and Welfare Clinical Trial and Research Center of Excellence (MOHW106-TDU-B-212-113004). The aforementioned institutes had no further role in study design; in the collection, analysis and interpretation of data; in the writing of the report; and in the decision to submit the paper for publication.

\section{- Conflicts of Interest}

No potential conflict of interest relevant to this article was reported.

\section{Author Contributions}

Conception and design, literature review, data interpretation, and manuscript writing: Chieh-Hsin Lin and Hsien-Yuan Lane. Participants enrollment: Chieh-Hsin Lin, Chih-Chiang Chiu, and Hsien-Yuan Lane. All authors

reviewed and approved the manuscript.

\section{ORCID}

Chieh-Hsin Lin

Chih-Chiang Chiu https://orcid.org/0000-0002-5475-230X

Hsien-Yuan Lane https://orcid.org/0000-0003-2162-8174

\section{REFERENCES}

1. Holtzman DM, Morris JC, Goate AM. Alzheimer's disease: the challenge of the second century. Sci Transl Med 2011;3: 77 sr 1 .

2. Levey A, Lah J, Goldstein F, Steenland K, Bliwise D. Mild cognitive impairment: an opportunity to identify patients at high risk for progression to Alzheimer's disease. Clin Ther 2006; 28:991-1001.

3. Sullivan PF, Fan C, Perou CM. Evaluating the comparability of gene expression in blood and brain. Am J Med Genet $B$ Neuropsychiatr Genet 2006;141B:261-268.

4. Cardinali DP, Brusco LI, Liberczuk C, Furio AM. The use of melatonin in Alzheimer's disease. Neuro Endocrinol Lett 2002;23 Supp/ 1:20-23.

5. Altun A, Ugur-Altun B. Melatonin: therapeutic and clinical utilization. Int J Clin Pract 2007;61:835-845.

6. Hardeland R. Antioxidative protection by melatonin: multiplicity of mechanisms from radical detoxification to radical avoidance. Endocrine 2005;27:119-130.

7. Ishio S, Yamada H, Craft CM, Moriyama Y. HydroxyindoleO-methyltransferase is another target for L-g/utamate-evoked inhibition of melatonin synthesis in rat pinealocytes. Brain Res 1999:850:73-78.

8. Yamada H, Yatsushiro S, Ishio S, Hayashi M, Nishi T, Yamamoto A, et al. Metabotropic glutamate receptors negatively regulate melatonin synthesis in rat pinealocytes. J Neurosci 1998;18:2056-2062.

9. Villela D, Atherino VF, Lima Lde S, Moutinho AA, do Amaral FG, Peres R, et al. Modulation of pineal melatonin synthesis by glutamate involves paracrine interactions between pinealocytes and astrocytes through NF- $\kappa B$ activation. Biomed Res Int 2013;2013:618432.

10. Bavithra S, Sugantha Priya E, Selvakumar K, Krishnamoorthy $\mathrm{G}$, Arunakaran J. Effect of melatonin on g/utamate: BDNF signaling in the cerebral cortex of polychlorinated biphenyls (PCBs)-exposed adult male rats. Neurochem Res 2015:40: 1858-1869.

11. Juan WS, Huang SY, Chang CC, Hung YC, Lin YW, Chen TY, et al. Melatonin improves neuroplasticity by upregulating the growth-associated protein-43 (GAP-43) and NMDAR postsynaptic density-95 (PSD-95) proteins in cultured neurons exposed to g/utamate excitotoxicity and in rats subjected to transient focal cerebral ischemia even during a long-term recovery period. J Pineal Res 2014;56:213-223.

12. Zhou JN, Liu RY, Kamphorst W, Hofman MA, Swaab DF. Early 
neuropathological Alzheimer's changes in aged individuals are accompanied by decreased cerebrospinal fluid melatonin levels. J Pineal Res 2003;35:125-130.

13. Srinivasan V, Pandi-Perumal SR, Cardinali DP, Poeggeler B, Hardeland R. Melatonin in Alzheimer's disease and other neurodegenerative disorders. Behav Brain Funct 2006;2:15.

14. Liu RY, Zhou JN, van Heerikhuize J, Hofman MA, Swaab DF. Decreased melatonin levels in postmortem cerebrospinal fluid in relation to aging, Alzheimer's disease, and apolipoprotein E-epsilon4/4 genotype. J Clin Endocrinol Metab 1999; 84:323-327.

15. Uchida K, Okamoto N, Ohara K, Morita Y. Daily rhythm of serum melatonin in patients with dementia of the degenerate type. Brain Res 1996;717:154-159.

16. Cardinali DP, Furio AM, Brusco LI. Clinical aspects of melatonin intervention in Alzheimer's disease progression. Curr Neuropharmacol 2010;8:218-227.

17. Cardinali DP, Furio AM, Brusco LI. The use of chronobiotics in the resynchronization of the sleep/wake cycle. Therapeutical application in the early phases of Alzheimer's disease. Recent Pat Endocr Metab Immune Drug Discov 2011;5:80-90.

18. McCleery J, Cohen DA, Sharpley AL. Pharmacotherapies for sleep disturbances in dementia. Cochrane Database Syst Rev 2016;11:CD009178.

19. Fourtillan JB, Brisson AM, Fourtillan M, Ingrand I, Decourt JP, Girault J. Melatonin secretion occurs at a constant rate in both young and older men and women. Am I Physiol Endocrinol Metab 2001;280:E11-E22.

20. Lu PH, Edland SD, Teng E, Tingus K, Petersen RC, Cummings JL. Donepezil delays progression to $A D$ in $\mathrm{MCl}$ subjects with depressive symptoms. Neurology 2009;72:2115-2121.

21. McKhann G, Drachman D, Folstein M, Katzman R, Price D, Stadlan EM. Clinical diagnosis of Alzheimer's disease: report of the NINCDS-ADRDA Work Group under the auspices of Department of Health and Human Services task force on Alzheimer's disease. Neurology 1984;34:939-944.

22. Morris JC. The Clinical Dementia Rating (CDR): current version and scoring rules. Neurology 1993;43:2412-2414.

23. Folstein MF, Folstein SE, McHugh PR. "Mini-mental state”. A practical method for grading the cognitive state of patients for the clinician. J Psychiatr Res 1975;12:189-198.

24. Crum RM, Anthony JC, Bassett SS, Folstein MF. Populationbased norms for the Mini-Mental State Examination by age and educational level. JAMA 1993;269:2386-2391.

25. Tombaugh TN, McIntyre NJ. The mini-mental state examination: a comprehensive review. J Am Geriatr Soc 1992;40:922935.

26. Lim WS, Chong MS, Sahadevan S. Utility of the clinical dementia rating in Asian populations. Clin Med Res 2007;5:6170.

27. Rockwood K, Strang D, MacKnight C, Downer R, Morris JC. Interrater reliability of the Clinical Dementia Rating in a multicenter trial. J Am Geriatr Soc 2000;48:558-559.
28. Hughes CP, Berg L, Danziger WL, Coben LA, Martin RL. A new clinical scale for the staging of dementia. Br J Psychiatry 1982;140:566-572.

29. Sack RL, Lewy AJ, Erb DL, Vollmer WM, Singer CM. Human melatonin production decreases with age. J Pineal Res 1986; 3:379-388.

30. Grad BR, Rozencwaig R. The role of melatonin and serotonin in aging: update. Psychoneuroendocrinology 1993; 18:283-295.

31. Srinivasan V, Kaur C, Pandi-Perumal S, Brown GM, Cardinali DP. Melatonin and its agonist ramelteon in Alzheimer's disease: possible therapeutic value. Int I Alzheimers Dis 2010; 2011:741974.

32. $\mathrm{Wu} \mathrm{YH}$, Swaab DF. The human pineal gland and melatonin in aging and Alzheimer's disease. J Pineal Res 2005;38:145-152.

33. Hardeland R, Pandi-Perumal SR, Cardinali DP. Melatonin. Int J Biochem Cell Biol 2006;38:313-316.

34. Cagnacci A. Melatonin in relation to physiology in adult humans. J Pineal Res 1996;21:200-213.

35. Ohashi Y, Okamoto N, Uchida K, lyo M, Mori N, Morita Y. Differential pattern of the circadian rhythm of serum melatonin in young and elderly healthy subjects. Biol Signals 1997; 6:301-306.

36. Scholtens RM, van Munster BC, van Kempen MF, de Rooij SE. Physiological melatonin levels in healthy older people: a systematic review. J Psychosom Res 2016;86:20-27.

37. Terzieva DD, Mateva ND, Vladimirova-Kitova LG. Melatonin reference limits at 3:00 AM and 8:00 AM in healthy adults. Clin Lab 2009;55:359-361.

38. Ji LD, Xu J, Wu DD, Xie SD, Tang NL, Zhang YP. Association of disease-predisposition polymorphisms of the melatonin receptors and sunshine duration in the global human populations. J Pineal Res 2010;48:133-141.

39. Obayashi K, Saeki K, Iwamoto J, Okamoto N, Tomioka K, Nezu S, et al. Positive effect of daylight exposure on nocturnal urinary melatonin excretion in the elderly: a cross-sectional analysis of the HEIJO-KYO study. J Clin Endocrinol Metab 2012;97:4166-4173.

40. Kasper S, Hajak G, Wulff K, Hoogendijk WJ, Montejo AL, Smeraldi $\mathrm{E}$, et al. Efficacy of the novel antidepressant agomelatine on the circadian rest-activity cycle and depressive and anxiety symptoms in patients with major depressive disorder: a randomized, double-blind comparison with sertraline. J Clin Psychiatry 2010;71:109-120.

41. Owen RT. Ramelteon: profile of a new sleep-promoting medication. Drugs Today (Barc) 2006;42:255-263.

42. Romero A, Egea J, García AG, López MG. Synergistic neuroprotective effect of combined low concentrations of galantamine and melatonin against oxidative stress in $\mathrm{SH}-\mathrm{SY} 5 \mathrm{Y}$ neuroblastoma cells. J Pineal Res 2010;49:141-148.

43. Feng Z, Chang Y, Cheng Y, Zhang BL, Qu ZW, Qin C, et al. Melatonin alleviates behavioral deficits associated with apoptosis and cholinergic system dysfunction in the APP 695 transgenic mouse model of Alzheimer's disease. J Pineal Res 
2004;37:129-136.

44. Ramos E, Egea J, de Los Ríos C, Marco-Contelles J, Romero A. Melatonin as a versatile molecule to design novel multitarget hybrids against neurodegeneration. Future Med Chem 2017; 9:765-780

45. Torres-Lista V, Parrado-Fernández C, Alvarez-Montón I, Frontiñán-Rubio J, Durán-Prado M, Peinado JR, et al. Neophobia, NQO1 and SIRT1 as premorbid and prodromal indicators of $A D$ in 3xTg-AD mice. Behav Brain Res 2014;271:140-146.

46. Reiter RJ, Mayo JC, Tan DX, Sainz RM, Alatorre-Jimenez M, Qin L. Melatonin as an antioxidant: under promises but over delivers. J Pineal Res 2016;61:253-278.

47. Rizzo V, Porta C, Moroni M, Scoglio E, Moratti R. Determination of free and total (free plus protein-bound) melatonin in plasma and cerebrospinal fluid by high-performance liquid chromatography with fluorescence detection. J Chromatogr B Analyt Technol Biomed Life Sci 2002;774:17-24.

48. Olakowska E, Marcol W, Kotulska K, Lewin-Kowalik J. The role of melatonin in the neurodegenerative diseases. Bratisl Lek Listy 2005;106:171-174.
49. Srinivasan V, Pandi-Perumal SR, Maestroni GJ, Esquifino AI, Hardeland R, Cardinali DP. Role of melatonin in neurodegenerative diseases. Neurotox Res 2005;7:293-318.

50. Lahiri DK, Chen DM, Lahiri P, Bondy S, Greig NH. Amyloid, cholinesterase, melatonin, and metals and their roles in aging and neurodegenerative diseases. Ann N Y Acad Sci 2005; 1056:430-449.

51. Wongprayoon P, Govitrapong P. Melatonin as a mitochondrial protector in neurodegenerative diseases. Cell Mol Life Sci 2017;74:3999-4014.

52. Song J, Whitcomb DJ, Kim BC. The role of melatonin in the onset and progression of type 3 diabetes. Mol Brain 2017;10:35.

53. Wu YH, Swaab DF. The human pineal gland and melatonin in aging and Alzheimer's disease. J Pineal Res 2005;38:145-152.

54. Lin CH, Chen PK, Chang YC, Chuo LJ, Chen YS, Tsai GE, et al. Benzoate, a D-amino acid oxidase inhibitor, for the treatment of early-phase Alzheimer disease: a randomized, doubleblind, placebo-controlled trial. Biol Psychiatry 2014;75:678685. 